\title{
Heat Transfer in a Tubular Reforming Catalyst Bed: Analytical Modelling
}

\author{
Mohammed W. Abdulrahman ${ }^{1}$ \\ ${ }^{1}$ Rochester Institute of Technology \\ Dubai Silicon Oasis, Dubai, UAE \\ mwacad@rit.edu
}

\begin{abstract}
Heat transfer in a reforming catalyst bed can play a crucial role in determining performance of such systems. The temperature distributions and heat transfer rates of tubular packed beds under steady state condition are important for proper prediction and control of the packed beds performance. This paper reports an analytical model of the steady-state heat transfer behaviour of a gas flowing through a packed bed under the constant wall temperature conditions. The steady state temperature distributions and heat transfer rates are derived by transforming the non-homogeneous partial differential energy equation into ordinary and homogeneous partial differential equations that are solved by the solution of an ordinary differential equation and separation of variables method. The final analytical solution of the steady state temperature distribution and heat transfer rate can be used as a continuous map for the moderator, which is highly preferred on using discrete numbers that are calculated from numerical analysis. The effect of system parameters such as; superficial velocity and solid particle diameter are also examined in this paper. By comparing with the pervious experimental works, the analytical model predicts the axial and radial temperature distributions quite well.
\end{abstract}

Keywords: heat transfer; reforming catalyst bed; mathematical modeling; separation of variables; temperature distribution; analytical modeling

\section{Introduction}

Since hydrogen is a clean energy fuel, different types of hydrogen energy systems have been explored. Steam reforming of fossil fuels is considered as the most utilized hydrogen supplying system in the industries. When a reactive fluid flows through a catalytically active packed bed, complex chemical and physical phenomena take place on different scales of the reactor. Due to the strong non-linearity of the reaction rate expressions in packed beds, an analytical solution of the system of differential equations can rarely be obtained. Therefore, the focus is usually on the numerical, approximate, solution of the equations. Many different numerical models for wall-cooled tubular reactors have been developed over the past years. The simplest model is one-dimensional and contains an overall heat transfer coefficient $(U)$ based on the difference between radial average temperature of particle bed and the corresponding wall temperature. A more complicated approach is the use of two-dimensional models with either a plug flow or an axially dispersed plug flow assumption. Depending on the temperature difference between packed particles and flowing fluid, models can be classified into one-phase homogeneous models and two-phase heterogeneous models $[1,2]$.

Some researchers have investigated the two-dimensional homogeneous models to predict the temperature profile in packed beds if the wall-fluid heat transfer coefficient and the effective radial thermal conductivity are used as the adjustable parameters $[3,4,5,6]$. These models, however, fail to represent quantitatively experimental results in many cases if independently determined heat transfer parameters are used $[7,8,9,10]$.

Radial temperature distribution at the inlet and outlet regions of packed beds have been measured by many published studies, such as, Schertz and Bischoff [11], Marivoet et al. [12], Lerou and Froment [13], Dixon [3], Freiwald and Paterson [14], Ziòlkowska and Ziòlkowski [15], Dixon and van Dongeren [4] and Nijemeisland and Dixon [6]. Dongsheng et al. [16] have performed experiments to study both the transient and steady-state heat transfer behaviour of a gas flowing through a packed bed under the constant wall temperature conditions. Abdulrahman [17, 18, 19, 20, 21] has reported experimental studies for the hydrodynamic and direct contact heat transfer in a slurry bubble column using a high temperature gas injected through a lower temperature slurry. He has formulated empirical equations for the gas holdup and the volumetric heat transfer coefficient for both bubbly and churn-turbulent flow regimes in addition to specifying the transition velocity between both 
regimes. Yutaka et al. [22] have investigated heat and mass transfer characteristics in a reforming catalyst bed experimentally. The effects of space velocity, steam carbon molar ratio, wall temperature, bed temperature, and catalyst particle diameter on the transport phenomena with chemical reaction were determined.

Bert Koning [23] has modelled the heat and mass transfer inside the tubular catalyst bed. His work is devoted to the resolution of the observed discrepancy between the heat transfer parameters obtained from experiments performed under reacting and non-reacting conditions. Iordanidis [24] has compared the predictions of wave models and the conventional standard dispersion models SDM for several industrial processes in order to indicate the range of applicability of the models and to identify the most important parameters affecting the differences between the models. Jong et al. [25] have modelled the natural gas conversion process within the reformer by both chemical reaction and heat transfer models. Abdulrahman $[26,27]$ has investigated the volumetric heat transfer coefficient and the axial temperature distribution of the direct contact heat transfer in slurry bubble column reactors, by using two dimensional CFD simulations. He has studied the effects of superficial gas velocity, static liquid height, and solid particle concentration on the volumetric heat transfer coefficient and the temperature profile. In his study, he has assumed that the slurry inside the slurry bubble column is a homogeneous mixture.

It can be seen from previous literatures that most of the mathematical models of the heat transfer in tubular packed bed reactors with chemical reactions, were analysed numerically. However, a heat transfer problem involving heat transfer by conduction and convection with chemical reactions and two dimensional cylindrical coordinates with boundary conditions of the type considered in this study, is presumably not solved analytically in the existing literature on the subject of packed bed systems. In this paper, the method of separation of variables is applied in order to develop expression for the temperature distribution as a function of axial and radial spaces.

The method of separation of variables can only be applied to one dimensional transient problems where both spatial boundary conditions are homogeneous. In the problems of tubular packed bed reactors with chemical reactions, this will not be the case and therefore more advanced techniques are required. This paper uses a method for breaking two dimensional steady state problems with non-homogeneous terms into sub-problems that can be solved either by separation of variables or by the solution of an ordinary differential equation. The closed-form analytical model can be used to derive operating maps that would help to identify the effects of heat transfer parameters on axial and radial temperature profiles.

\section{Theoretical Development}

In this paper, it should be noted that the term "packed bed reactor" means a single tube packed with catalytic particles. However, in industrial cooled or heated packed bed reactors a bundle of tubes filled with catalyst is usually arranged within a large reactor shell. A fluid circulating in the exterior of the tubes removes or supplies heat to the packed tubes.

The most often two dimensional heat balance model is the pseudo-homogeneous model, which is given in the form of non-dimensional parameters, by;

$$
P e \frac{\partial \theta}{\partial \bar{z}}-\frac{k_{z}}{k_{r}} \frac{\partial^{2} \theta}{\partial \bar{z}^{2}}=\frac{\partial^{2} \theta}{\partial \bar{r}^{2}}+\frac{1}{\bar{r}} \frac{\partial \theta}{\partial \bar{r}}+\operatorname{Pe} S Q_{T},
$$

with the following dimensionless boundary conditions;

$$
\left.\frac{\partial \theta}{\partial \bar{r}}\right|_{\bar{r}=0}=0,\left.\frac{\partial \theta}{\partial \bar{r}}\right|_{\bar{r}=1}=-\frac{h_{w}}{k_{r}} R_{t} \theta, \theta(\bar{r}, 0)=1,\left.\quad \frac{\partial \theta}{\partial \bar{z}}\right|_{\bar{z}=\frac{L}{R_{t}}}=0,
$$

where; 


$$
\theta=\frac{T-T_{w}}{T_{i}-T_{w}} \quad, \quad \bar{z}=\frac{z}{R_{t}} \quad, \quad \bar{r}=\frac{r}{R_{t}},
$$

and;

$$
P e_{r}=\frac{u \rho C_{p} R_{t}}{K_{r}} \quad, \quad S=\frac{R_{t}}{u \rho C_{p}\left(T_{i}-T_{w}\right)} \quad, \quad Q_{T}=-\Delta H_{r} R_{i}
$$
and $\left.\theta_{z}\right)$.

It is necessary to split the solution $(\theta)$ into a homogeneous $\left(\theta_{h}\right)$ and one or more non-homogeneous components $\left(\theta_{r}\right.$

$$
\theta(\bar{r}, \bar{z})=\theta_{h}(\bar{r}, \bar{z})+\theta_{r}(\bar{r})
$$

By substituting Eq. (5), into Eq. (1), and enforcing a homogeneous partial differential equation for the homogeneous component of the solution, this will lead to an ordinary differential equation for $\theta_{r}$ with the solution;

$$
\theta_{r}=\frac{\operatorname{Pe} S Q_{T} k_{r}}{4 h_{w}}\left[\frac{2}{R_{t}}+\frac{h_{w}}{k_{r}}\left(1-\bar{r}^{2}\right)\right]
$$

and a homogeneous partial differential equation for $\theta_{h}$ where the solution can be expressed as;

$$
\theta_{h}=\theta_{h r} \theta_{h z}
$$

Substituting Eq. (7) into the homogeneous partial differential equation for $\theta_{h}$ and using Sturm-Liouville system of an ordinary differential equation in the direction with the homogeneous boundary conditions, leads to the two ordinary differential equations;

$$
\theta_{h r}{ }^{\prime \prime}+\frac{1}{\bar{r}} \theta_{h r}{ }^{\prime}+\lambda^{2} \theta_{h r}=0
$$

and,

$$
\frac{k_{z}}{k_{r}} \theta_{h z}{ }^{\prime \prime}-P e \theta_{h z}{ }^{\prime}-\lambda^{2} \theta_{h z}=0
$$

The solution of Eq. (8) is;

$$
\theta_{h r n}\left(\lambda_{n} \bar{r}\right)=A_{3 n} J_{0}\left(\lambda_{n} \bar{r}\right) \quad \text { where } \quad n=1,2,3, \ldots \ldots, \infty
$$

where; $\theta_{h r n}$ are referred to as eigen functions, and the values $\lambda_{n}$ are the eigenvalues associated with each eigen function.

With the eigen problem solved, it is necessary to return to the non-homogeneous portion of the problem, $\theta_{h z}$. Each of the eigenvalues is associated with an ordinary differential equation in the $\bar{z}$-direction according to Eq. (9).

According to Eq. (7), the solution for $\theta_{h}$ associated with the $n$th eigenvalue is;

$$
\theta_{h n}=C_{1 n} e^{\frac{1 k_{r}}{2 k_{z}}\left(P e+\sqrt{P e^{2}+4 \frac{k_{z}}{k_{r}} \lambda_{n}^{2}}\right) \bar{z}} J_{0}\left(\lambda_{n} \bar{r}\right)+C_{2 n} e^{\frac{1 k_{r}}{2 k_{z}}\left(P e-\sqrt{P e^{2}+4 \frac{k_{z}}{k_{r}} \lambda_{n}^{2}}\right) \bar{z}} J_{0}\left(\lambda_{n} \bar{r}\right)
$$


where $\quad n=1,2,3, \ldots \ldots, \infty$, and $C_{1 n}$ and $C_{1 n}$ are undetermined constants.

Because the partial differential equation, Eq. (1), is linear, the sum of the solutions for each eigenvalue, $\theta_{h n}$ given by Eq. (11), is itself a solution; $\theta_{h}=\sum_{n=1}^{\infty} \theta_{h n}$.

Substituting this equation into the boundary condition of $\left.\theta_{h}\right|_{\bar{z}=0}$ leads to;

$$
\sum_{n=1}^{\infty}\left(C_{1 n}+C_{2 n}\right) J_{0}\left(\lambda_{n} \bar{r}\right)=1-\theta_{r}
$$

Orthogonality property of eigen functions can be used to determine the constants $C_{n}$. By using the Sturm-Liouville system, to get orthogonality, Eq. (12) must be multiplied by $\bar{r} J_{0}\left(\lambda_{m} \bar{r}\right)$ and integrated from $\bar{r}=0$ to $\bar{r}=1$, to get an equation that relate $C_{1 n}$ and $C_{2 n}$. Another equation can be obtained between $C_{1 n}$ and $C_{2 n}$, by substituting the solution of $\theta_{h}$ into the boundary condition of, $\left.\frac{\partial \theta_{h}}{\partial \bar{z}}\right|_{\bar{z}=\frac{L}{R}}=0$, and then using the orthogonality property again. By solving the two equations that relate the constants $C_{1 n}$ and $C_{2 n}$, these constants can be obtained as;

$$
\begin{gathered}
C_{1 n}=0 \\
C_{2 n}=\frac{\frac{P e S Q_{T}}{\lambda_{n}^{2}} J_{0}\left(\lambda_{n}\right)+\frac{1}{\lambda_{n}} J_{1}\left(\lambda_{n}\right)\left(1-\operatorname{Pe} S Q_{T}\left(\frac{k_{r}}{h_{w} R_{t}}+\frac{2}{\lambda_{n}^{2}}\right)\right)}{\frac{1}{2}\left(J_{0}^{2}\left(\lambda_{n}\right)+J_{1}^{2}\left(\lambda_{n}\right)\right)}
\end{gathered}
$$

And the solution of $T$ is;

$$
T=T_{w}+\left(T_{i}-T_{w}\right)\left[\sum_{n=1}^{\infty}\left[C_{2 n} e^{\frac{1 k_{r}}{2 k_{z}}\left(P e-\sqrt{P e^{2}+4 \frac{k_{z}}{k_{r}} \lambda_{n}^{2}}\right) \bar{z}} J_{0}\left(\lambda_{n} \bar{r}\right)\right]+\frac{P e S Q_{T} k_{r}}{4 h_{w}}\left[\frac{2}{R_{t}}+\frac{h_{w}}{k_{r}}\left(1-\bar{r}^{2}\right)\right]\right]
$$

Eq. (14) is the equation that expresses the steady state temperature distribution in the tubular reforming catalyst bed as a function of radial and axial distances, with a heat generation (or consumption) per unit reactor volume of $\left(Q_{T}\right)$.

In the calculation of heat transfer flux, since the temperature difference between the tube outer surface and the inner surface is small, it can be neglected. Gaseous thermo physical properties are assessed at mean temperature (fluid temperature is assumed to be equal to particle temperature) and mean composition within the catalyst bed. Heat flux can be expressed as;

$$
q=h_{w}\left(T_{w}-T\right)
$$

\section{Results and Discussion}

In this section, the theoretical analysis is applied to the experimental work of Dongsheng et al. (2006) [16], whose work was part of a catalytic reacting system for non-periodic adsorption enhanced chemical reaction process for low-temperature hydrogen production. They have performed experiments that include steady state axial and radial temperature distributions at different Reynolds numbers and the results of these experiments are compared with the analytical results of this paper. Also, heat flux distributions of a packed bed reactor system are investigated for both axial and radial directions. Moreover, 
the effects of Reynolds number $(R e)$, heat generation per unit reactor volume $\left(Q_{T}\right)$ and particle size $\left(d_{p}\right)$ on the temperature distribution and heat flux are examined. The data used in the modeling of the above system are given in Table 1.

Table 1: Reactor geometry and operating conditions used in the simulation of the above analysis.

\begin{tabular}{|c|l|c|}
\hline Material & Parameter description & Data \\
\hline Stainless steel cylindrical reactor & Reactor length $(L)$ & $1.1 \mathrm{~m}$ \\
\cline { 2 - 3 } & Inside diameter $\left(d_{i}\right)$ & $0.041 \mathrm{~m}$ \\
\cline { 2 - 3 } & Outside diameter $\left(d_{o}\right)$ & $0.048 \mathrm{~m}$ \\
\cline { 2 - 3 } & Wall Temperature $\left(T_{w}\right)$ & $100^{\circ} \mathrm{C}$ \\
\hline Glass balls & Particle diameter $\left(d_{p}\right)$ & $0.005 \mathrm{~m}$ \\
\hline Air gas & Inlet temperature $\left(T_{i}\right)$ & $20^{\circ} \mathrm{C}$ \\
\hline
\end{tabular}

\subsection{Axial Temperature and Heat Flux Distributions of the Catalyst Layer}

Fig. 2a shows the steady state temperature distribution with the axial distance $(z)$ for different radial locations $(r)$. As seen in this figure, at a specified radial position, the temperature rise is increased in the upstream of the catalyst bed and is gradually decreased at the downstream of the bed. By comparing the curves in Fig. 2a, it can be seen that the temperature increase rate is higher near the wall than that in the center. It is obvious, that the reason of this, is the effect of heat convection at the wall $\left(r=R_{t}\right)$.

The distribution of heat flux with the axial distance is shown in Fig. 2b. A higher heat flux is found at the upstream of the tube and the heat flux is gradually decreased along the gas stream. This behavior means that the most part of the reforming reaction is performed at the upstream of the tube. It can be seen from Fig. 2b, that the distribution of heat flux is affected by that of the temperature, because heat transfer coefficient and wall temperature are considered constants in this analysis. This explains why the heat flux near the wall is lower than that at the center of the catalyst bed.

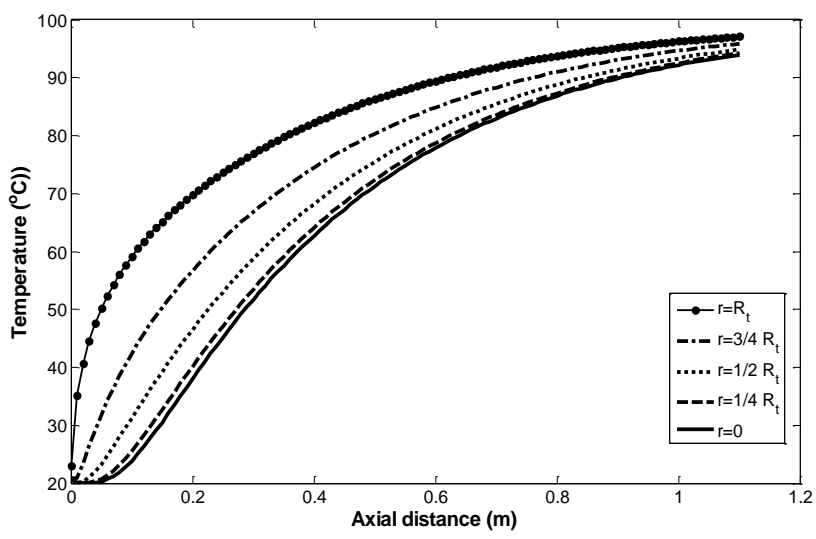

(a)

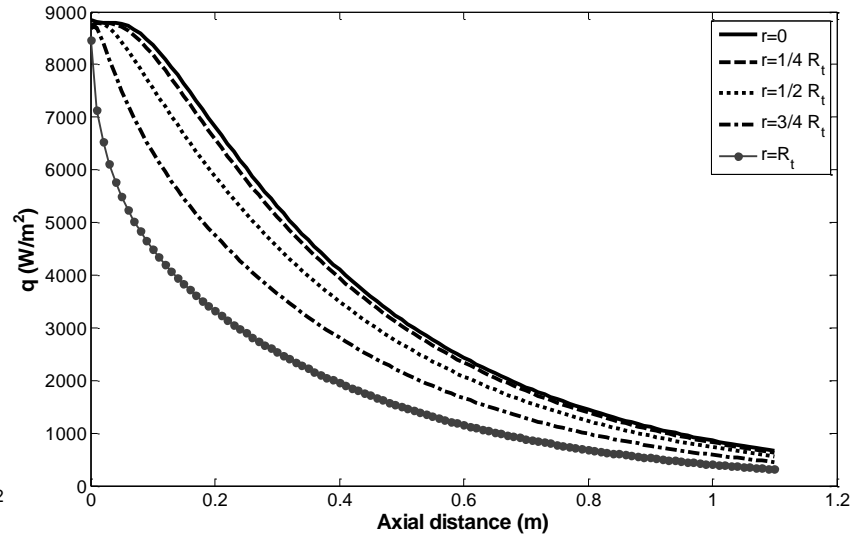

(b)

Fig. 2: Steady state axial temperature and heat flux distributions at different radii $(R e=556)$.

\subsection{Radial Temperature and Heat Flux Distributions of the Catalyst Layer}

Fig. 3a shows the radial temperature distribution at the steady state with different axial positions. As seen in this figure, at a specified axial position, the temperature increases near the wall and gets minimum at the centre of the reactor. This tendency is due to the radial heat convection at the wall of the tube where the temperature is constant. It is also shown that the radial temperature distribution is higher at the downstream of the reactor. A comparison between the radial temperature distributions in Fig. 3a indicates that the non-uniformity of the radial temperature distribution decreases with increasing the axial distance. 
Fig. 3b shows the radial heat flux distribution with different axial positions. It shows that the heat transfer rate at the center is higher than that at the wall. Also, it is shown that the heat transfer rate is low and more uniform at the downstream of the catalyst bed and is enhanced with more non-uniformity in the upstream of the bed.

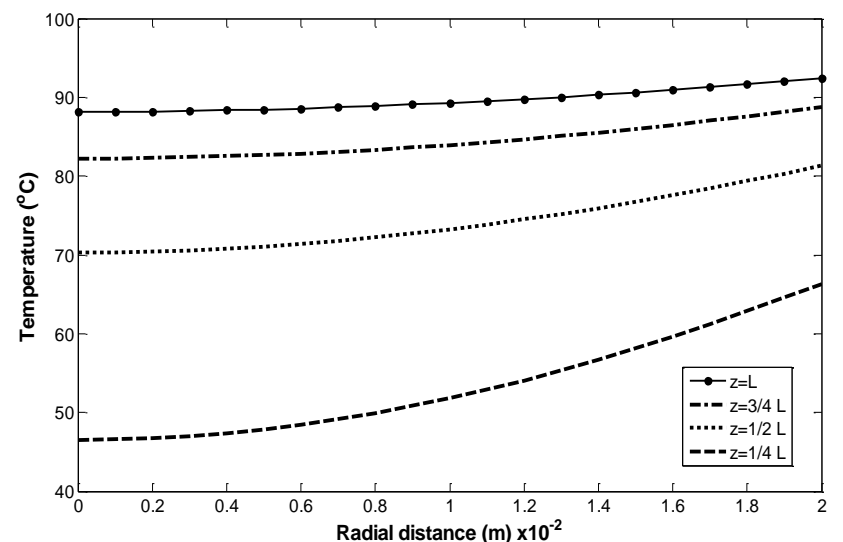

(a)

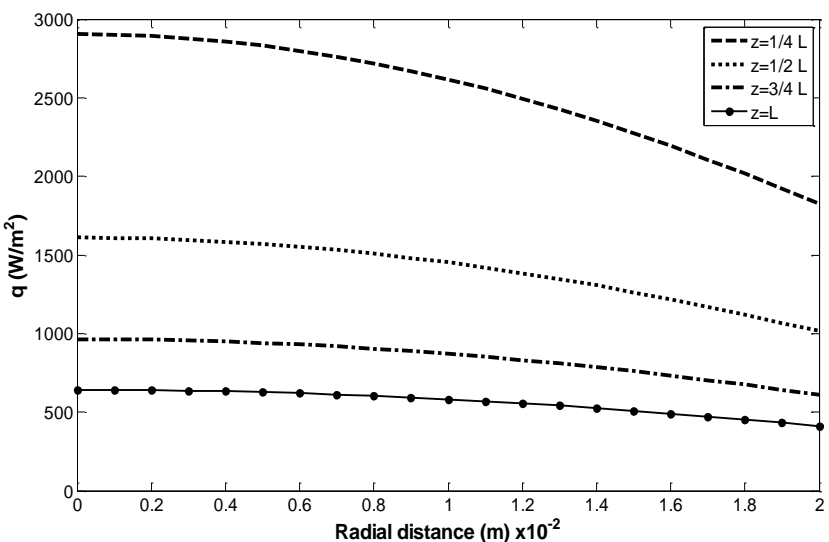

(b)

Fig. 3: Steady state radial temperature and heat flux distributions at different axial positions $(R e=556)$.

\subsection{Comparison with Published Works}

The axial and radial temperature distributions of present model have been compared with those of experimental work by Dongsheng et al. (2006) [16] at different Reynolds numbers as shown in Fig. 8 and 9. Dongsheng et al. studied the transient and steady-state heat transfer behaviour of a gas flowing through a packed bed under the constant wall temperature conditions. The analytical solution shows a good agreement with Dongsheng work for different Reynolds numbers. The maximum percentage difference between the two axial temperature profiles is less than $6 \%$ when $R e=187$, and between the two radial temperature profiles is less than $1.2 \%$ when $R e=291$. This is due to the use of non-exact correlations of effective transport parameters.
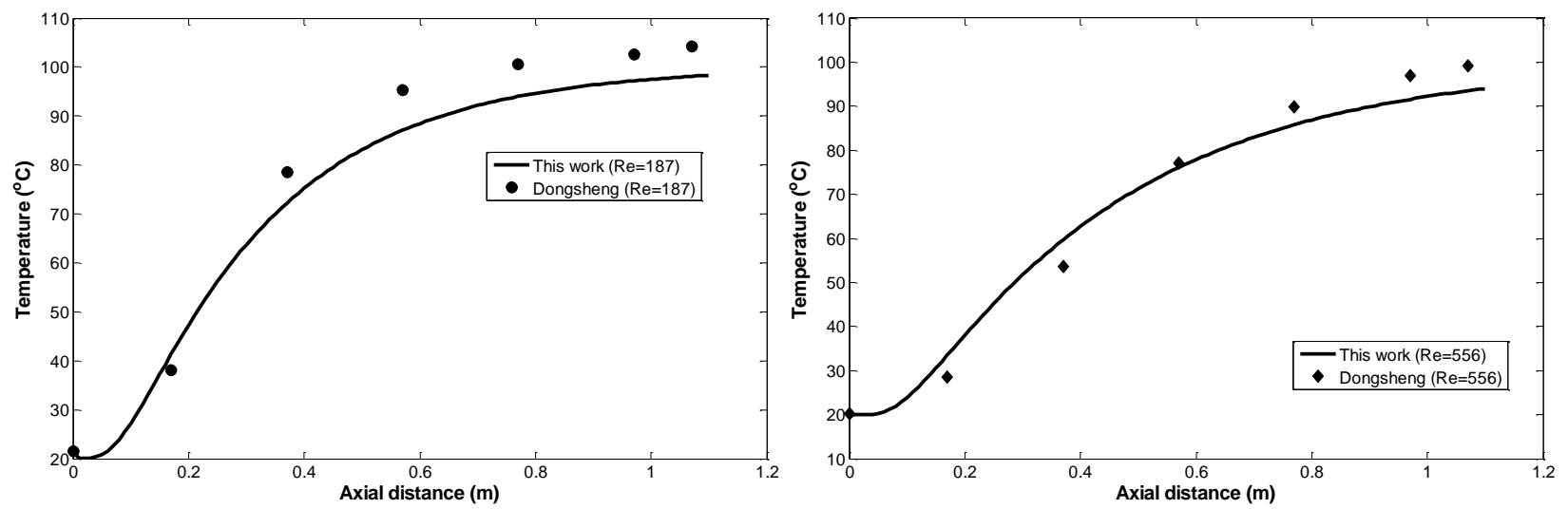

Fig. 8: Comparison of axial temperature distribution of present model with the experimental work of Dongsheng at the centre of the reactor. 


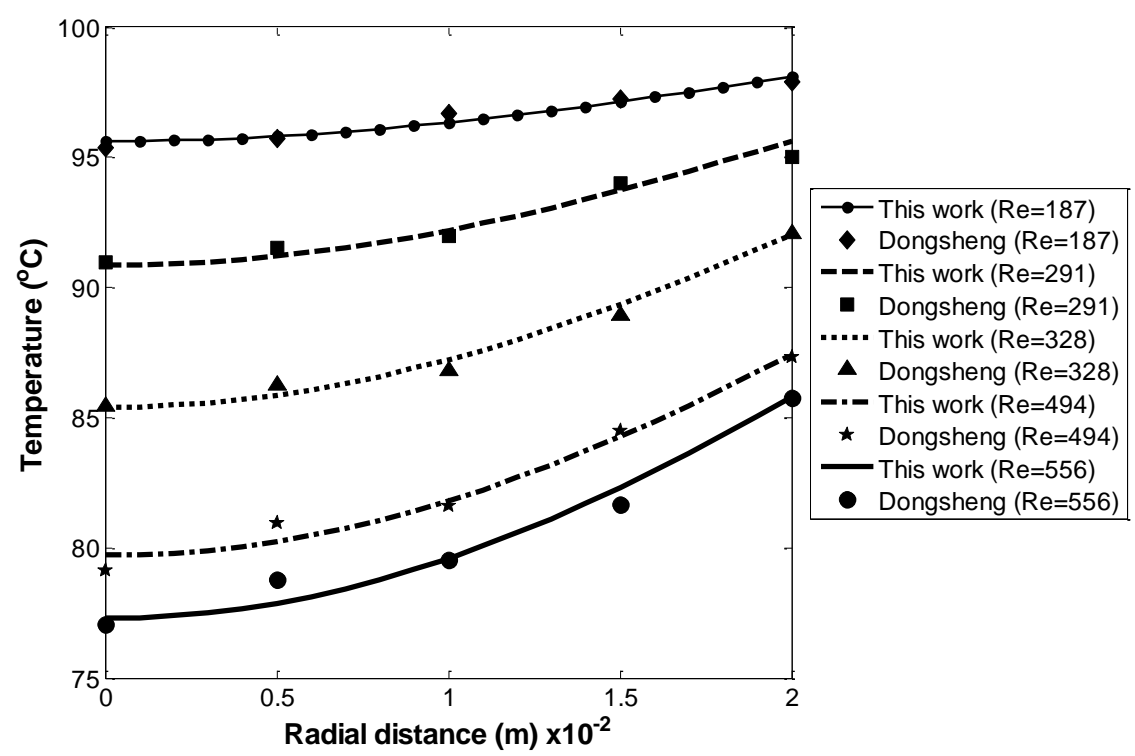

Fig. 9: Comparison of radial temperature distributions of this model with the experimental work of Dongsheng at different Reynolds numbers $(z=579 \mathrm{~mm})$.

\section{Conclusions}

In this paper, steady-state heat transfer behaviour of a gas flowing through a packed bed has been investigated analytically. The method of separation of variables was applied to the steady state energy equation in cylindrical coordinates, in order to provide a simplified formulation that can be used to identify the temperature distribution and the heat transfer rate. Both radial and axial direction temperature distributions have been calculated under the constant wall temperature conditions. Effective thermal conductivities and convective heat transfer coefficient have been used in the analysis. By comparing with the previous experimental results, the analytical model predicts the axial and radial temperature distributions quite well. The model of this work can be used for many types of tubular flow that have heat transfer with or without chemical reactions. The solutions presented in this study can be useful in the verification of the two-dimensional numerical codes of packed beds.

\section{Nomenclature}

$\begin{array}{cl}C_{p} & \text { Specific heat, } \mathrm{J} / \mathrm{Kg} . \mathrm{K} \\ h_{w} & \text { Wall heat transfer coefficient, } \mathrm{W} / \mathrm{m} .{ }^{\circ} \mathrm{C} \\ k_{z} & \text { Effective axial conductivity, } \mathrm{W} / \mathrm{m} .{ }^{\circ} \mathrm{C} \\ k_{r} & \text { Effective radial conductivity, } \mathrm{W} / \mathrm{m} .{ }^{\circ} \mathrm{C} \\ P e & \text { Heat transfer Peclet number in radial direction } \\ r & \text { Radial distance, } \mathrm{m} \\ R_{i} & \text { Reaction rate, } \mathrm{mole} / \mathrm{Kg} . \mathrm{s} \\ R_{t} & \text { Tube radius, } \mathrm{m} \\ T & \text { Temperature, }{ }^{\circ} \mathrm{C} \\ T_{i} & \text { Inlet Temperature, }{ }^{o} \mathrm{C} \\ T_{w} & \text { Wall temperature, }{ }^{o} \mathrm{C} \\ u & \text { Superficial velocity, } \mathrm{m} / \mathrm{s} \\ z & \text { Axial distance, } m \\ \Delta H_{r} & \text { Reaction enthalpy, } \mathrm{J} / \mathrm{mole} \\ \rho & \text { Fluid density, } \mathrm{Kg} / \mathrm{m}^{3}\end{array}$




\section{References}

[1] A. P. De Wasch and G. F. Froment, "A two dimensional heterogeneous model for fixed bed catalytic reactors," Chemical Engineering Science, vol. 26, pp. 629-634, 1971.

[2] A. P. De Wasch and G. F. Froment, "Heat transfer in packed beds," Chemical Engineering Science, vol. 27, pp. 567$576,1972$.

[3] A. G. Dixon, "The length effect on packed bed effective heat transfer parameters," Chemical Engineering Journal, vol. 31, pp. 163-173, 1985.

[4] A. G. Dixon and J. H. van Dongeren, "The influence of the tube and particle diameters at constant ratio on heat transfer in packed beds," Chemical Engineering and Processing, vol. 37, pp. 23-32, 1998.

[5] Y. Demirel, R. N. Sharma and H. H. Al-Ali, "On the effective heat transfer parameters in a packed bed," International Journal of Heat and Mass Transfer, vol. 43, pp. 327-332, 2000.

[6] M. Nijemeisland and A. G. Dixon, "Comparison of CFD simulations to experiment for convective heat transfer in a gas-solid fixed bed," Chemical Engineering Journal, vol. 82, pp. 231-246, 2001.

[7] W. R. Paterson and J. J. Carberry, "Fixed bed catalytic reactor modelling: the heat transfer problem," Chemical Engineering Science, vol. 38, pp. 175-180, 1983.

[8] G. Eigenberger and W. Ruppel, "Problems of mathematical modelling of industrial fixed-bed reactors," German Chemical Engineering, vol. 9, pp. 74-83, 1986.

[9] T. Daszkowski and G. Eigenberger, "A re-evaluation of fluid flow, heat transfer and chemical reaction in catalyst filled tubes," Chemical Engineering Science, vol. 47, pp. 2245-2250, 1992.

[10] O. Bey and G. Eigenberger, "Gas flow and heat transfer through catalyst filled tubes," International Journal of Thermal Sciences, vol. 40, pp. 152-164, 2001.

[11] W. W. Schertz and K. B. Bischoff, "Thermal and material transport in non-isothermal packed beds," Journal of American Institute of Chemical Engineers, vol. 15, pp. 597-604, 1969.

[12] J. Marivoet, P. Teodoroiu and S. J. Wajc, "Porosity, velocity and temperature profiles in cylindrical packed beds," Chemical Engineering Science, vol. 29, pp. 1836-1840, 1974.

[13] J. J. Lerou and G. F. Froment, "Velocity, temperature and conversion profiles in fixed bed catalytic reactors," Chemical Engineering Science, vol. 32, pp. 853-861, 1977.

[14] M. G. Freiwald and W. R. Paterson, "Accuracy of model predictions and reliability of experimental data for heat transfer in packed beds," Chemical Engineering Science, vol. 47, pp. 1545-1560, 1992.

[15] I. Ziòlkowska and D. Ziòlkowski, "Modelling of gas interstitial velocity radial distribution over a cross-section of a tube packed with a granular catalyst bed," Chemical Engineering Science, vol. 48, pp. 3283-3292, 1993.

[16] W. Dongsheng, D. Yulong, "Heat transfer of gas flow through a packed bed," Chemical Engineering Science, vol. 61, pp. $3532-3542,2006$.

[17] M. W. Abdulrahman, "Experimental studies of direct contact heat transfer in a slurry bubble column at high gas temperature of a helium-water-alumina system, Applied Thermal Engineering, vol. 91, pp. 515-524, 2015.

[18] M. W. Abdulrahman, "Experimental studies of gas holdup in a slurry bubble column at high gas temperature of a helium- water- alumina system," Chemical Engineering Research and Design, vol. 109, pp. 486-494, 2016.

[19] M. W. Abdulrahman, "Experimental studies of the transition velocity in a slurry bubble column at high gas temperature of a helium-water-alumina system," Experimental Thermal and Fluid Science, vol. 74, pp. 404-410, 2016.

[20] M. W. Abdulrahman, "Direct contact heat transfer in the thermolysis reactor of hydrogen production cu-cl cycle," US Patent 10, 059, 586, August 28, 2018.

[21] M. W. Abdulrahman, "Analysis of the thermal hydraulics of a multiphase oxygen production reactor in the $\mathrm{Cu}-\mathrm{Cl}$ cycle," Ph.D. dissertation, Dept. Mech. Eng., UOIT, Oshawa, ON.

[22] U. Yutaka, F. Shoichiro and Y. Masahiko, "Heat and mass transfer in a reforming catalyst bed: quantitative evaluation of the controlling factor by experiment," Journal of Thermal Science and Technology, vol. 2, no. 2, pp. 146-155, 2007. 
[23] K. Bert, Heat and mass transport in tubular packed bed reactors at reacting and non-reacting conditions. Twente University Press, 2002.

[24] A. A. Iordanidis, Mathematical Modeling of Catalytic Fixed Bed Reactors. Twente University Press, 2002.

[25] M. De Jong, A.H.M.E. Reinders, J.B.W. Kok and G. Westendorp, “Optimizing a steam methane reformer for hydrogen production," International Journal of Hydrogen Production, vol. 34, pp. 285-292, 2009.

[26] M. W. Abdulrahman, "CFD simulations of direct contact volumetric heat transfer coefficient in a slurry bubble column at a high gas temperature of a helium-water-alumina system," Applied Thermal Engineering, vol. 99, pp. 224-234, 2016.

[27] M. W. Abdulrahman, "CFD Analysis of Temperature Distributions in a Slurry Bubble Column with Direct Contact Heat Transfer," in Proceedings of the 3rd International Conference on Fluid Flow, Heat and Mass Transfer (FFHMT'16), Ottawa, ON, 2016.

[28] N. Wakao, S. Kaguei and H. Nagai, "Effective diffusion coefficients for fluid species reacting with first order kinetics in packed bed reactors and discussion on evaluation of catalyst effectiveness factors," Chemical Engineering Science, vol. 33, pp. 183-187, 1978. 